United Nations Educational Scientific and Cultural Organization

and

International Atomic Energy Agency

THE ABDUS SALAM INTERNATIONAL CENTRE FOR THEORETICAL PHYSICS

\title{
SOLITONS OF SCALAR FIELD WITH INDUCED NONLINEARITY AND THEIR STABILITY
}

Bijan Saha*

Bogoliubov Laboratory of Theoretical Physics, Joint Institute for Nuclear Research, Dubna, 141980, Moscow region, Russian Federation ${ }^{\dagger}$

and

The Abdus Salam International Centre for Theoretical Physics, Trieste, Italy.

\begin{abstract}
Exact particle-like static, spherically and/or cylindrically symmetric solutions to the equations of interacting scalar and electromagnetic field system have been obtained. We considered FRW and Gödel universes as external gravitational field with spherical and cylindrical symmetry respectively. Beside the usual solitons some special regular solutions known as droplets, anti-droplets and hats (confined in finite interval and having trivial value beyond it) have been obtained. It has been shown that in FRW space-time equations with different interaction terms may have stable solutions while within the scope of Gödel model only the droplet-like and the hat-like configurations may be stable, providing that they are located in the region where $g^{00}>0$.
\end{abstract}

MIRAMARE - TRIESTE

September 1999

\footnotetext{
*E-mail: saha@thsun1.jinr.ru

†Permanent address.
} 


\section{INTRODUCTION}

The concept of a soliton as a stable particle-like state of nonlinear system has so caught the imagination of physicists and mathematicians of all descriptions that it can genuinely claim to be one of the few interdisciplinary subjects of modern day mathematical physics $[1,2]$. One of the fields to apply the soliton concept is the elementary particle physics, where the soliton solutions of nonlinear field equations are used as the simplest models of extended particles [3,4]. Development of general relativity (GR) and quantum field theory (QFT) leads to the increasing interest to study the role of gravitational field in elementary particle physics. To obtain and study the properties of regular localized solutions to the nonlinear classical field equations is motivated mainly by a hope to create a consistent, divergence-free theory. These solutions, as was remarked by Rajaraman [5] give us one of the ways of modeling elementary particles as extended objects with complicated spatial structure. In such attempts it is natural to treat the field nonlinearity not only as a tool for avoiding the theoretical difficulties (e.g., singularities) but also as a reflection of real properties of physical systems. It should be emphasized that the complete description of elementary particles with all their physical characteristics (e.g., magnetic momentum) can be given only within the framework of interacting field theory [6]. Let us remark that the choice of the field equations is one of the principle problems in nonlinear theory. At present there is no criterion for the selection of interaction Lagrangian and any Lorentz-invariant combination of field functions can be considered as such Lagrangian.

Recently, solitons with spherical and/or cylindrical symmetry of the interacting system of massless scalar, electromagnetic and gravitational fields given by the Lagrangian

$$
L=\frac{R}{2 \kappa}-\frac{1}{16 \pi} F_{\alpha \beta} F^{\alpha \beta}+\frac{1}{8 \pi} \varphi, \alpha \varphi^{, \alpha} \Psi(I)
$$

where $\kappa=8 \pi G$ is the Einstein gravitational constant and the function $\Psi(I)$ of the invariant $I=A_{\mu} A^{\mu}$ characterizes the interaction between the scalar $\varphi$ and electromagnetic $A_{\mu}$ fields, have been obtained by us [7]. Unfortunately, the stability of these solutions is yet to be determined. Even the study of stability of solitons of scalar field with induced nonlinearity (given by the Lagrangian (2.1)) is rather complicated if the proper gravitational field is taken into account. For this reason we will consider an interacting system of scalar and electromagnetic and external gravitational fields, confining ourselves to static and spherically and/or cylindrically symmetric configurations. For simplicity we will consider Friedmann-Robertson-Walker and Gödel models describing spherically and cylindrically-symmetric space-time, respectively. Within the framework of these simple models, it will be shown that though the (external) gravitational field is a minor player in formation of soliton-like solutions, it can play a crucial role through symmetry when one deals with the stability problem. 


\section{FUNDAMENTAL EQUATIONS}

We consider the nonlinear generalization of the theory that is related to the introduction of direct interaction between neutral scalar and electromagnetic fields. The decay process like $\pi^{0} \rightarrow 2 \gamma$, described by the effective Lagrangian [8]

$$
L_{\mathrm{int}}=\varphi_{\pi^{0}} F_{\alpha \beta} F^{* \alpha \beta},
$$

indicates the possibility of such generalization. Thus we consider a system with the Lagrangian

$$
L=\frac{1}{2} \varphi_{, \alpha} \varphi^{, \alpha}-\frac{1}{16 \pi} F_{\alpha \beta} F^{\alpha \beta} \Psi(\varphi)
$$

where $\Psi(\varphi)$ is some arbitrary function characterizing the interaction between the scalar $(\varphi)$ and electromagnetic $\left(F_{\mu \nu}\right)$ fields, takes the form

$$
\Psi(\varphi)=1+\lambda \Phi(\varphi)
$$

As is seen, for $\lambda=0, \Psi(\varphi) \equiv 1$ and we have the system with minimal coupling. Note that the Lagrangian (2.1) describes the system of fields with positive definite energy if $\Psi(\varphi) \geq$ 0 . This kind of interaction has been thoroughly discussed in [9]. Note that this type of interaction gives rise to induced nonlinearity which is crucial for stability. Let us write the scalar and the electromagnetic field equations corresponding to the Lagrangian(2.1)

$$
\begin{gathered}
\frac{1}{\sqrt{-g}} \frac{\partial}{\partial x^{\nu}}\left(\sqrt{-g} g^{\nu \mu} \frac{\partial \varphi}{\partial x^{\mu}}\right)+\frac{1}{16 \pi} F_{\alpha \beta} F^{\alpha \beta} \Psi_{\varphi}=0, \quad \Psi_{\varphi}=\frac{\partial \Psi}{\partial \varphi} \\
\frac{1}{\sqrt{-g}} \frac{\partial}{\partial x^{\nu}}\left(\sqrt{-g} F^{\nu \mu} \Psi(\varphi)\right)=0
\end{gathered}
$$

The corresponding energy-momentum tensor reads

$$
T_{\mu}^{\nu}=\varphi_{, \mu} \varphi^{, \nu}-\frac{1}{4 \pi} F_{\mu \beta} F^{\nu \beta} \Psi(\varphi)-\delta_{\mu}^{\nu}\left[\frac{1}{2} \varphi_{, \alpha} \varphi^{, \alpha}-\frac{1}{16 \pi} F_{\alpha \beta} F^{\alpha \beta} \Psi(\varphi)\right]
$$

\section{SPHERICALLY SYMMETRIC CONFIGURATIONS}

\section{A. Solutions in FRW Universe}

As an external homogeneous and isotropic gravitational field we choose the FRW space-time. This Universe is very important as the corresponding cosmological models coincide with observation. The interval in the FRW Universe, in general, takes the form $[10,11]$ 


$$
d s^{2}=d t^{2}-R^{2}(t)\left[\frac{d r^{2}}{1-k r^{2}}+r^{2}\left\{d \vartheta^{2}+\sin ^{2} \vartheta d \phi^{2}\right\}\right]
$$

Here $R(t)$ defines the size of the Universe, and $k$ takes the values 0 and \pm 1 . In the static case, that is for $R=$ const. $k=0$ corresponds to usual Minkowski space, $k=+1$ describes the close Einstein Universe [12] and $k=-1$ corresponds to the space-time with hyperbolic spatial cross-section. Note that the velocity of light $c$ has been taken to be unity.

As was mentioned earlier, we seek the static, spherically symmetric solutions to the equations (2.2) and (2.3) assuming that the scalar field is the function of $r$ only, i.e. $\varphi=\varphi(r)$. We also assume that the electromagnetic field possesses only one component $F_{10}=\partial A_{0} / \partial r$.

Under the assumption made above, the solution for equation (2.3) reads

$$
F^{01}=\bar{q} P(\varphi) \frac{\sqrt{1-k r^{2}}}{R^{3} r^{2}}
$$

where $\bar{q}$ is the constant of integration and $P(\varphi)=1 / \Psi(\varphi)$. Putting (3.2) into (2.2) for the scalar field we obtain the equation with "induced nonlinearity" [13,14]

$$
\left(1-k r^{2}\right) \varphi^{\prime \prime}+\frac{2-3 k r^{2}}{r} \varphi^{\prime}-\frac{2 q^{2}}{R^{2} r^{4}} P_{\varphi}=0, \quad q^{2}=\frac{\bar{q}^{2}}{16 \pi}
$$

Substituting $z=\sqrt{1 / r^{2}-k}$ we can rewrite equations (3.2) and (3.3) in the form

$$
\begin{gathered}
\frac{\partial A_{0}}{\partial z}=\frac{\bar{q}}{R} P(\varphi) \\
\frac{\partial^{2} \varphi}{\partial z^{2}}-\frac{2 q^{2}}{R^{2}} P_{\varphi}=0
\end{gathered}
$$

The first integral of (3.5) reads

$$
\frac{\partial \varphi}{\partial z}=\frac{2 q}{R} \sqrt{P+C_{0}}
$$

Here $C_{0}$ is the constant of integration, which under the regularity condition of $T_{0}^{0}$ at the center turns to be trivial, i.e., $C_{0}=0$. Finally, we write the solution of the scalar field equation in quadrature

$$
\int \frac{\partial \varphi}{\sqrt{P}}=\frac{2 q}{R}\left(z-z_{0}\right)
$$

In accordance with (3.2) and (3.6) from (2.4) we find the density of field energy of the system

$$
T_{0}^{0}=\frac{4 q^{2} P}{R^{4} r^{4}}
$$


and total energy of the material field system

$$
E_{f}=\int T_{0}^{0} \sqrt{-{ }^{3} g} d^{3} \mathbf{x}=-8 \pi q \int \sqrt{P} d \varphi
$$

Thus, we see that the energy density $T_{0}^{0}$ and total energy $E_{f}$ of the configurations obtained do not depend on the conventional values of the parameter $k$. As one sees, to write the scalar $\varphi$ and electromagnetic $A_{0}$ functions as well as the energy density $\left(T_{0}^{0}\right)$ and energy of the material fields $\left(E_{f}\right)$ explicitly, one has to give $P(\varphi)$ in an explicit form. As was mentioned earlier, the function $P(\varphi)$ is an arbitrary one. The concrete choice of $P(\varphi)$ depends on what one is looking for. Here we will consider $P(\varphi)$ in the form that provides soliton-like solutions to the nonlinear scalar field equation.

Let us choose $P(\varphi)$ in the form

$$
P(\varphi)=P_{0} \cos ^{2}\left(\frac{\lambda \varphi}{2}\right)
$$

with $\lambda$ being the interaction parameter. Inserting (3.10) into (3.5) we get the sin-Gordon type equation [15]

$$
\frac{\partial^{2} \varphi}{\partial z^{2}}+\frac{\lambda q^{2} P_{0}}{4 R^{2}} \sin (\lambda \varphi)=0
$$

with the solution

$$
\varphi(z)=\frac{2}{\lambda} \arcsin \tanh \left[b\left(z+z_{1}\right)\right], \quad b=\frac{\lambda q \sqrt{P_{0}}}{R}, \quad z_{1}=\text { const }
$$

Let us analyze the solution (3.12). It can be shown that $\lim _{r \rightarrow 0} \varphi=\pi / \lambda$ for all $k$. As one sees, the solution possesses meaning only in the region where $z=\sqrt{1 / r^{2}-k}>0$. It means that, in the case of $k=+1$ the configuration confines in the interval $0 \leq r \leq 1$. Then the asymptotic behavior of the solution (3.12) can be written as

$$
\varphi \rightarrow \begin{cases}0, k=-1, \quad z_{1}=-1, \quad r \rightarrow \infty \\ 0, \quad k=0, \quad z_{1}=0, \quad r \rightarrow \infty \\ 0, \quad k=+1, \quad z_{1}=0, \quad r \geq 1\end{cases}
$$

The electromagnetic field in this case reads

$$
A_{0}=\frac{\bar{q} P_{0}}{b R} \tanh \left[b\left(z+z_{1}\right)\right]
$$

As one sees, $\lim _{r \rightarrow 0} A_{0}=\bar{q} P_{0} / b R$ for all $k$ and $A_{0} \rightarrow 0$ as $r \rightarrow \infty$ if we choose the constant $z_{1}$ as was done for scalar field.

From (3.9) we find the total energy of the system $E_{f}=-16 \pi q \sqrt{P_{0}} / \lambda$.

Choosing $P(\varphi)$ in the form 


$$
P=\lambda\left(a^{2}-\varphi^{2}\right)^{2}
$$

with $\lambda$ being the coupling constant and $a$ being some arbitrary constant, from (3.5) we obtain

$$
4 \lambda a^{2} \varphi-4 \lambda \varphi^{3}+\frac{\partial^{2} \varphi}{\partial z^{2}}=0
$$

The equation (3.16) can be seen as an MKdV one. Indeed, a KdV equation

$$
\frac{\partial u}{\partial t}+\alpha u^{p} \frac{\partial u}{\partial x}+\beta \frac{\partial^{3} u}{\partial x^{3}}=0
$$

can always be converted to

$$
-D u+\alpha \frac{u^{p+1}}{p+1}+\beta \frac{d^{2} u}{d z^{2}}=0
$$

if one looks for a stationary solution of the form $u=u(z)$ where $z=x-D t$. In our particular case $p=2$ and equation (3.16) is an MKdV one. The scalar field function in this case has the form [16]

$$
\varphi(z)=a \tanh \left[\sqrt{\lambda} a b\left(z+z_{2}\right)\right], \quad b=\frac{2 q}{R}
$$

Taking into account that $z=\sqrt{1 / r^{2}-k}$ one sees that at the origin $\lim _{r \rightarrow 0} \varphi=a$ whereas at the asymptotic region for different value of $k$ we get

$$
\varphi \rightarrow \begin{cases}0, k=-1, \quad z_{2}=-1, & r \rightarrow \infty \\ 0, & k=0, \quad z_{2}=0, \quad r \rightarrow \infty \\ 0, & k=+1, \quad z_{2}=0, \quad r \geq 1\end{cases}
$$

The electromagnetic field in this case reads

$$
A_{0}=\frac{\bar{q} \lambda a^{4}}{b R}\left\{\tanh \left[b\left(z+z_{2}\right)\right]-(1 / 3) \tanh ^{3}\left[b\left(z+z_{2}\right)\right]\right\}
$$

Analyzing as earlier we conclude that $\lim _{r \rightarrow 0} A_{0}=\bar{q} \lambda a^{4} / b R$ and as $r \rightarrow \infty, A_{0} \rightarrow 0$ if we choose the constant $z_{2}$ as above.

From (3.9) we find the total energy of the system to be $E_{f}=-16 \pi q \sqrt{\lambda} a^{3} / 3$.

A specific type of solution to the nonlinear field equations in flat space-time was obtained in a series of interesting articles [17]. These solutions are known as dropletlike solutions or simply droplets. The distinguishing property of these solutions is the availability of some sharp boundary defining the space domain in which the material field happens to be located, i.e., the field is zero beyond this area. It was found that the solutions mentioned exist in field theory with specific interactions that can be considered 
as an effective one, generated by initial interactions of unknown origin. In contrast to the widely known soliton-like solutions, with field functions and energy density asymptotically tending to zero at spatial infinity, the solutions in question vanish at a finite distance from the center of the system (in the case of spherical symmetry) or from the axis (in the case of cylindrical symmetry). Thus, there exists a sphere or cylinder with critical radius $r_{0}$ outside of which the fields disappear. Therefore the field configurations have a droplet-like structure $[13,17,18]$.

To obtain the droplet-like configuration we choose a very specific type of interaction function $P(\varphi)$ which has the form [19] [cf. FIG. 1]

$$
P(\varphi)=J^{2-4 / \sigma}\left(J^{2 / \sigma}-1\right)^{2}
$$

where $J=\lambda \varphi, \quad \sigma=2 n+1, \quad n=1,2 \cdots$. Putting (3.22) into (3.7) one gets

$$
\left|J^{2 / \sigma}-1\right|=\exp \left[ \pm \frac{4 q \lambda}{R \sigma}\left(z-z_{0}\right)\right]
$$

Let us consider the case when $\left|J^{2 / \sigma}-1\right|=1-J^{2 / \sigma}$. Taking the sign in exponent to be a "minus" one from (3.23) we obtain

$$
\varphi(z)=\frac{1}{\lambda}\left[1-\exp \left(-\frac{4 q \lambda}{R \sigma}\left(z-z_{0}\right)\right)\right]^{\sigma / 2}
$$

Recalling that $z=\sqrt{1 / r^{2}-k}$ from (3.24) we see that at $r \rightarrow 0$ the scalar field $\varphi$ takes the value $\varphi(0) \rightarrow 1 / \lambda$ and at $r \rightarrow r_{c}=1 / \sqrt{z_{0}^{2}+k}$, the scalar field function becomes trivial, i.e., $\varphi\left(r_{c}\right) \rightarrow 0$. It is obvious that for $r>r_{c}$ the value of the square bracket turns out to be negative and $\varphi(r)$ becomes imaginary, since $\sigma$ is an odd number. Since we are interested in real $\varphi$ only, without loss of generality we may assume the value of $\varphi$ is zero for $r \geq r_{c}$, matching at $r=r_{c}$ (i.e., $z=z_{0}$ ) being smooth [cf. FIG. 2]. Note that, for $k=+1$, the scalar field is confined in the region $0 \leq r \leq 1$, as it was in the previous two cases. The total energy of the droplet we obtain from (3.9) has the form

$$
E_{f}=\frac{4 \pi q}{\lambda(\sigma-1)}
$$

As is seen from (3.25), the value of the total energy does not depend on the size of the droplet, it means that droplets of different linear size share the same total energy. Let us now go back to (3.23) again and consider the sign in exponent to be "plus". In this case we find

$$
\varphi(z)=\frac{1}{\lambda}\left[1-\exp \left(\frac{4 q \lambda}{R \sigma}\left(z-z_{0}\right)\right)\right]^{\sigma / 2}
$$

Contrary to the droplet this configuration possesses a trivial value up to $r=r_{c}=$ $1 / \sqrt{z_{0}^{2}+k}$, then begins to increase taking maximum value at spatial infinity:

$$
\varphi=\frac{1}{\lambda}\left[1-\exp \left(\frac{4 q \lambda z_{0}}{R \sigma}\right)\right]^{\sigma / 2} \leq 1
$$


For the choice of $P(\varphi)$ in the form (3.22) we obtain

$$
A_{0}=\frac{\bar{q}}{4 q \lambda}\left\{\frac{J^{2-2 / \sigma}}{1-1 / \sigma}-J^{2}\right\}
$$

where $J=\lambda \varphi$. It can be shown that for this case $A_{0 \max }=\bar{q} / 4 q \lambda(\sigma-1)$ as $r \rightarrow 0$ and $A_{0}=0$ for $r \geq r_{c}$ [cf. FIG. 3], while for the anti-droplets $A_{0}$ is trivial at $r \leq r_{c}$ and $A_{0 \max } \leq \bar{q} / 4 q \lambda(\sigma-1)$ at spatial infinity.

Note that the total energy of the anti-droplet (3.26) is equal to that of the droplet.

Finally, we consider another very interesting case putting

$$
P(\varphi)=\frac{\sigma^{2}}{4} Q^{2-4 / \sigma}\left(A^{2}-4 Q^{2 / \sigma}\right)
$$

where $Q=R \varphi / 2 q, \quad \sigma=2 n+1, \quad n=1,2 \cdots$. Putting (3.28) into (3.7) one gets

$$
\varphi(z)=\frac{2 q}{R}[(z-a)(b-z)]^{\sigma / 2}
$$

where we put $A=b-a$ and $2 z_{0}=b+a$. As one sees this configuration is completely localized in the interval $r \in\left(1 / \sqrt{b^{2}+k}, 1 / \sqrt{a^{2}+k}\right)$ [cf. FIG. 4]. The expression for $A_{0}$ in this case is a very complicated one, though it can be expressed by elementary functions. The total energy of this configuration is trivial.

Thus, we obtain different particle-like configurations for different choices of the interaction function $P(\varphi)$. As one can see, all these solutions are totally determined by the nonlinear term (interaction function) in the equation regardless if space-time is flat or curved.

\section{B. Stability problem}

To study the stability of the configurations obtained we write the linearized equations for the radial perturbations of scalar field assuming that

$$
\varphi(r, t)=\varphi(r)+\xi(r, t), \quad \xi \ll \varphi
$$

Here once again we note that our main object of investigation is the scalar field, while the electromagnetic field is the auxiliary one generating induced nonlinearity in scalar field equation. So we consider only a small perturbation of scalar field leaving the electromagnetic one unaltered. Putting (3.30) into (2.2) in view of (3.3) we get for $\xi(r, t)$ the equation

$$
\ddot{\xi}+3 \frac{\dot{R}}{R} \dot{\xi}-\frac{1-k r^{2}}{R^{2}} \xi^{\prime \prime}-\frac{2-3 k r^{2}}{r R^{2}} \xi^{\prime}+\frac{q^{2} P_{\varphi \varphi}}{R^{4} r^{4}} \xi=0
$$


As the external gravitational field is a cosmological one, we can consider $R(t)$ as a slowly varying time-function: $\dot{R} \approx 0$. Assuming that

$$
\xi(r, t) \approx v(r) \exp (-i \Omega t), \quad \Omega=\omega / R
$$

from (3.31) we obtain

$$
\left(1-k r^{2}\right) v^{\prime \prime}-\frac{2-3 k r^{2}}{r} v^{\prime}+\left[\omega^{2}-\frac{q^{2} P_{\varphi \varphi}}{R^{2} r^{4}}\right] v=0
$$

The substitution

$$
\eta(\zeta)=r \cdot v(r), \quad \zeta=\frac{1}{\sqrt{k}} \arcsin (\sqrt{k} r)
$$

leads equation (3.33) to the Liouville one [20]

$$
\eta_{\zeta \zeta}+\left(\omega^{2}-V(\varphi)\right) \eta=0
$$

with the potential

$$
V(\varphi)=-k+\frac{q^{2} P_{\varphi \varphi}}{R^{2} \zeta^{4}}\left(\frac{\sqrt{k} \zeta}{\sin (\sqrt{k} \zeta)}\right)^{4}
$$

The system considered here is instable if there exists physically permissible perturbations increasing infinitely at time. From (3.32) follows that $\xi$ may increase exponentially if $\omega^{2}<$ 0 . So that the perturbations were physically allowable, they should be finite and regular at the center $(r=0)$ and vanish at spatial infinity $(r \rightarrow \infty)$. For the regular configuration of fields the boundary condition at the center is $|\xi|<\infty$, whence it follows that $\eta=0$ at $r=0$. At the spatial infinity physically allowable perturbations should satisfy the condition $\eta<\infty$, whence for fixed $t, \xi \rightarrow 0$ at $r \rightarrow \infty$. Thus, the stability problem is reduced to detecting the stationary states with negative energy for one-dimensional quantum mechanical system given by (3.35) with the potential (3.36). So far the quantity $\omega^{2}$ plays the role of energy, the existence of stationary quantum states is the necessary and sufficient condition of instability for the type of perturbations considered. And to clarify for which values of $\omega^{2}$ there exist stationary states, it is necessary to study the potential $V$ (3.36). For the potential $V \geq 0$ for $0 \leq r<\infty$ we have the stable solutions. Here it should be emphasized that, the condition $V \geq 0$ is neither the necessary nor the sufficient condition of stability, since some potential wells $(V<0$ for some value of $r$ ) may not contain states with negative energy.

For the interaction term (3.10) we see that

$$
V(\varphi)>0, \quad \text { if and only if } \tanh ^{2} b\left(z+z_{1}\right)>\frac{k \zeta^{4} R^{2}}{P_{0} q^{2} \lambda^{2}}\left(\frac{\sin (\sqrt{k} \zeta)}{\sqrt{k} \zeta}\right)^{4}+\frac{1}{2}
$$


Thus we find that the equations with trigonometric nonlinearity contain stable solutions. Given $P(\varphi)$ in the form (3.15) we find that

$$
V(\varphi)>0, \quad \text { if } \quad \tanh ^{2} b\left(z+z_{2}\right)>\frac{k \zeta^{4} R^{2}}{12 q^{2} \lambda}\left(\frac{\sin (\sqrt{k} \zeta)}{\sqrt{k} \zeta}\right)^{4}+\frac{a^{2}}{3}
$$

As is seen from (3.38) the equations with polynomial type of nonlinearity too contain

some stable solutions. For the droplet-like configurations, i.e., for the interacting term $P(\varphi)$ given by $(3.22)$, it can be shown that the potential

$$
\lim _{r \rightarrow 0} V(\varphi) \rightarrow+\infty, \quad \lim _{r \rightarrow r_{c}} V(\varphi) \rightarrow+\infty
$$

beginning with $\sigma \geq 5$. That means that the droplet-like configurations (3.24) with $\sigma \geq 5$ are stable for the class of perturbation, vanishing at $r=0$ and $r=r_{c}$. The same can be concluded for the solutions (3.26) and (3.29). As one can see from (3.36), the external gravitational field can strengthen or weaken the stability of the configurations obtained depending on the value of $k$.

\section{CYLINDRICALLY SYMMETRIC CONFIGURATIONS}

\section{A. Solutions in Gödel Universe}

In the previous section we studied the possibility of the formation of regular localized configuration in homogeneous and isotropic FRW Universes. Now let us continue our study in the homogeneous but anisotropic Universe. In particular, we consider the model proposed by Gödel. The linear element of the Gödel Universe in cylindrical coordinates reads [21]

$$
d s^{2}=d t^{2}-d \rho^{2}+\frac{1}{\Omega^{2}}\left[\sinh ^{4} \Omega \rho-\sinh ^{2} \Omega \rho\right] d \phi^{2}-\frac{\sqrt{8}}{\Omega} \sinh ^{2} \Omega \rho d \phi d t-d z^{2}
$$

where the constant $\Omega$ is related to the angular velocity $\omega: \omega=\sqrt{2} \Omega$. This form of linear element of the four-dimensional homogeneous space $S$ directly exhibits its rotational symmetry, since the $g_{\mu \nu}$ do not depend on $\phi$. It is easy to find

$$
\lim _{\Omega \rightarrow 0} \sqrt{-g}=\lim _{\Omega \rightarrow 0} \frac{1}{2 \Omega} \sinh (2 \Omega \rho) \rightarrow \rho
$$

i.e. at $\omega \rightarrow 0$ Gödel Universe transfers to the flat one.

As in the spherically symmetric case, here we also seek the static solutions of equations (2.2) and (2.3) assuming the scalar field to be the function of $\rho$ only, i.e., $\varphi=\varphi(\rho)$ and the electromagnetic possesses only one component $F_{13}=\partial A_{3} / \partial \rho$. For the electromagnetic field in this case we find 


$$
F^{13}=2 \Omega C_{1} P / \sinh (2 \Omega \rho), \quad C_{1}=\text { const }
$$

The scalar field equation (2.2) with regard to (4.2) reads

$$
\frac{\partial^{2} \varphi}{\partial \rho^{2}}+2 \Omega \operatorname{coth}(2 \Omega \rho) \frac{\partial \varphi}{\partial \rho}=\frac{8 q^{2} \Omega^{2} P_{\varphi}}{\sinh ^{2}(2 \Omega \rho)}, \quad q^{2}=C_{1}^{2} / 16 \pi
$$

Substituting $y=\frac{1}{2 \Omega} \ln \tanh (\Omega \rho)$ we rewrite (4.2) and (4.3) in the form

$$
\frac{\partial A_{3}}{\partial y}=2 \Omega C_{1} P
$$

and

$$
\frac{\partial^{2} \varphi}{\partial y^{2}}-8 q^{2} \Omega^{2} P_{\varphi}=0
$$

The first integral of (4.5) reads

$$
\frac{\partial \varphi}{\partial y}= \pm 4 q \Omega \sqrt{P+D_{0}}
$$

Here $D_{0}$ is the constant of integration, which under the regularity condition of $T_{0}^{0}$ at the center turns to be trivial, i.e., $D_{0}=0$. Finally, we write the solution of the scalar field equation in quadrature

$$
\int \frac{\partial \varphi}{\sqrt{P}}=4 q \Omega\left(y-y_{0}\right)
$$

In accordance with (4.2) and (4.6) from (2.4) we find the density of field energy and the total energy of the system

$$
\begin{aligned}
& T_{0}^{0}=\frac{16 q^{2} \Omega^{2} P}{\sinh ^{2}(2 \Omega \rho)} \\
& E_{f}=8 \pi q \int \sqrt{P} d \varphi
\end{aligned}
$$

As in the previous case, to write the scalar $(\varphi)$ and vector $(A)$ functions as well as the energy density $\left(T_{0}^{0}\right)$ and energy of the material fields $\left(E_{f}\right)$ explicitly, one has to give $P(\varphi)$ in an explicit form. Here again we will thoroughly study the solutions obtained for different concrete forms of $P(\varphi)$.

Choosing $P(\varphi)$ in the form (3.10), i.e.,

$$
P(\varphi)=P_{0} \cos ^{2}\left(\frac{\lambda \varphi}{2}\right)
$$

with $\lambda$ being the interaction parameter, from (4.5) we get the sin-Gordon type equation

$$
\frac{\partial^{2} \varphi}{\partial y^{2}}+4 \lambda q^{2} \Omega^{2} P_{0} \sin (\lambda \varphi)=0
$$


The solution to this equation can be written in the form

$$
\varphi(\rho)=\frac{4}{\lambda}\left[\arctan \left(\frac{\tanh \Omega \rho}{\Omega \rho_{0}}\right)^{\alpha}-\frac{\pi}{4}\right], \quad \alpha=\lambda q \sqrt{P_{0}}
$$

where $\rho_{0}$ is the constant of integration, giving the size of the system. Without loss of generality we can choose $\alpha>0$. Then one can find $\lim _{\rho \rightarrow 0} \varphi=-\pi / \lambda$. For $\rho>0$ the field $\varphi$ steadily increases up to $\pi / \lambda$. In particular, at spatial infinity we get $\lim _{\rho \rightarrow \infty} \varphi=0$. In this case $P\left(\varphi_{\infty}\right)=1$ which corresponds to the exclusion of interaction at spatial infinity. The total energy of the system in this case coincides with that of FRW Universe, i.e., $E_{f}=-16 \pi q \sqrt{P_{0}} / \lambda$. The electromagnetic field in this case has the form

$$
A_{3}=\frac{4 \Omega}{\lambda} \sqrt{\pi P_{0}} \sin (\lambda \varphi / 2)
$$

Choosing $P(\varphi)$ in the form (3.15), i.e.,

$$
P=\lambda\left(a^{2}-\varphi^{2}\right)^{2}
$$

from (4.5) we, as in the FRW case, again obtain MKdV type equation with the solution

$$
\varphi(\rho)=a \frac{\tanh ^{\alpha}(\Omega \rho)-1}{\tanh ^{\alpha}(\Omega \rho)+1}, \quad \alpha=4 a q \sqrt{\lambda}
$$

From (4.15) it is clear that $\lim _{\rho \rightarrow 0} \varphi \rightarrow-a$ and $\lim _{\rho \rightarrow \infty} \varphi \rightarrow 0$. From (4.9) we find the total energy of the system to be $E_{f}=-16 \pi q \sqrt{\lambda} a^{3} / 3$ as it was in the FRW case. In this case we get

$$
A_{3}=2 \Omega \sqrt{\pi \lambda}\left[a^{2} \varphi-\varphi^{3} / 3\right]
$$

The choice of the interaction term $P(\varphi)$ in the form (3.22), i.e.,

$$
P(\varphi)=J^{2-4 / \sigma}\left(1-J^{2 / \sigma}\right)^{2}
$$

where $J=\lambda \varphi, \quad \sigma=2 n+1, \quad n=1,2 \cdots$, leads to the following expression for scalar field

$$
\varphi(\rho)=\frac{1}{\lambda}\left[1-\left(\frac{\tanh (\Omega \rho)}{\tanh \left(\Omega \rho_{0}\right)}\right)^{\alpha}\right]^{\sigma / 2}, \quad \alpha= \pm \frac{4 \lambda q}{\sigma}
$$

where $\rho_{0}$ is an arbitrary constant. For $\alpha>0$ the solution possesses physical meaning at $\rho<\rho_{0}$ and becomes meaningless at $\rho>\rho_{0}$. Outside the cylinder $\rho=\rho_{0}$ one can put $\varphi \equiv 0$. This trivial solution is stitched with the solution at $\rho=\rho_{0}$ under condition $\varphi^{\prime}\left(\rho_{0}\right)=0$, which fulfills if and only if $4 \lambda|q|>\sigma$. Consequently, at $\rho>\rho_{0}$ the Lagrangian becomes physically meaningless, however its limiting value at $\rho \rightarrow \rho_{0}-0$ is equal to zero. Continuing it at $\rho>\rho_{0}$, one can consider the field to be totally trivial in this area. Thus 
we get the droplet-like configuration. The field $\varphi$ steadily decreases from $\varphi(0)=1 / \lambda$ to $\varphi\left(\rho_{0}\right)=0$ with $\varphi^{\prime}(0)=0$ (for $\sigma \geq 3$ ) and $\varphi^{\prime}\left(\rho_{0}\right)=0$. The total energy of the "droplet" is defined as

$$
E_{f}=\frac{4 \pi q}{\lambda(\sigma-1)}
$$

which remains unaltered even in flat space-time $(\Omega=0)$. It means that the "droplet" is not sensitive to Gödel gravitational field. On the other hand, for $\alpha<0$ we obtain the configuration (anti-droplet) possessing trivial value at $\rho \leq \rho_{0}$. Starting from $\rho=\rho_{0}$ it increases and at spatial infinity takes the maximum value: $\varphi(\infty) \leq 1 / \lambda$. As in the FRW case, electromagnetic field now takes the form

$$
A_{3}=\frac{\Omega \sqrt{p i}}{\lambda}\left(\frac{J^{2-2 / \sigma}}{1-1 / \sigma}-J^{2}\right)
$$

Finally we consider the case providing hat-like configuration. Putting

$$
P(\varphi)=\frac{\sigma^{2}}{4} Q^{2-4 / \sigma}\left(A^{2}-4 Q^{2 / \sigma}\right)
$$

where $Q=R / 4 q \Omega, \quad \sigma=2 n+1, \quad n=1,2 \cdots$. Inserting (4.21) into (4.7) one gets

$$
\varphi(y)=4 q \Omega[(y-a)(b-y)]^{\sigma / 2}
$$

where we put $A=b-a$ and $2 y_{0}=b+a$. As one can see, the configuration is completely localized in the interval $y \in(a, b)$ with trivial total energy.

\section{B. Stability problem}

Let us now study the stability of the configuration obtained. In doing so we consider the perturbed scalar field $\delta \varphi=\chi(\rho, t)$ that leaves the cylindrical-symmetry of the system unbroken. The linearized equation for the perturbed scalar field is

$$
\frac{1}{\sqrt{-g}} \frac{\partial}{\partial x^{\mu}}\left(\sqrt{-g} g^{\mu \nu} \chi_{, \nu}\right)+\frac{q^{2}}{2(-g)} P_{\varphi \varphi} \chi=0
$$

Since, for the case considered, $g^{00}=\left(1-\sinh ^{2} \Omega \rho\right) / \cosh ^{2} \Omega \rho$, it is clear that equation (4.23) changes on the surface where $\sinh \Omega \rho=1$. The Cauchy problem for (4.23) is incorrect by Hadamard in the region, where $g^{00}<0$. In connection with this, only the droplet-like and hat-like solutions can be stable by Lyapunov, if they are located in the region where $g^{00}>0$ [13]. Assuming that

$$
\chi(\rho, t)=v(\rho) \exp (-i \varepsilon t)
$$


from (4.23) we get

$$
v^{\prime \prime}+2 \Omega \operatorname{coth}(2 \Omega \rho) v^{\prime}+\left[\frac{1-\sinh ^{2} \Omega \rho}{\cosh ^{2} \Omega \rho} \varepsilon^{2}-\frac{2 \Omega^{2} q^{2}}{\sinh ^{2}(2 \Omega \rho)} P_{\varphi \varphi}\right] v=0
$$

The substitution

$$
\eta(\zeta)=\xi(\rho) \cdot v(\rho)
$$

where

$$
\zeta=\int \frac{\sqrt{1-\sinh ^{2} \Omega \rho}}{\cosh \Omega \rho} d \rho, \quad \xi=\left[4 \sinh ^{2} \Omega \rho\left(1-\sinh ^{2} \Omega \rho\right)\right]^{1 / 4}
$$

leads to the equation for perturbed field to the Liouville one

$$
\frac{\partial^{2} \eta}{\partial \zeta^{2}}+\left[\varepsilon^{2}-V\right] \eta=0
$$

Here the effective potential $V(\varphi)$ takes the form

$$
V(\varphi)=\frac{\Omega^{2}}{\left(1-\sinh ^{2} \Omega \rho\right)}\left\{\frac{q^{2} P_{\varphi \varphi}}{2 \sinh ^{2} \Omega \rho}+\frac{\left(4 \sinh ^{6} \Omega \rho-16 \sinh ^{4} \Omega \rho+3 \sinh ^{2} \Omega \rho-1\right) \cosh ^{2} \Omega \rho}{4 \sinh ^{2} \Omega \rho\left(1-\sinh ^{2} \Omega \rho\right)}\right\}
$$

For the interaction function, chosen in the form (3.22), one finds

$$
P_{\varphi \varphi}=\lambda^{2}\left(\frac{2 \sigma^{2}-12 \sigma+16}{\sigma^{2} J^{4 / \sigma}}-\frac{4 \sigma^{2}-12 \sigma+8}{\sigma^{2} J^{2 / \sigma}}+2\right)
$$

Taking into account that $\lim _{\rho \rightarrow 0} J \rightarrow 1$ and $\lim _{\rho \rightarrow \rho_{0}} J \rightarrow 0$, it can be shown that

$$
\begin{aligned}
& \lim _{\rho \rightarrow 0} V(\varphi) \rightarrow+\infty \quad \text { for } \quad \sigma<4 q \lambda \\
& \lim _{\rho \rightarrow \rho_{0}} V(\varphi) \rightarrow+\infty \quad \text { for } \quad \sigma \geq 5
\end{aligned}
$$

Here we used the fact that $g^{00}>0$, i.e., $\sinh \Omega \rho<|1|$. Thus, as in the previous case, we find that the droplet-like configurations are stable for $5 \leq \sigma<4|q| \lambda$. Analogically, we can conclude that the hat-like configurations are also stable beginning with $\sigma \geq 5$. Here it should be emphasized that, contrary to the droplets (anti-droplets) where $\sigma$ may be big enough, in the case of hat-like solutions $\sigma$ should be small. Otherwise $\varphi$ itself may be large. Nevertheless, one can always choose $P$ in such a way, e.g.,

$$
P=(1 / 4)(\sigma Q)^{2-4 / \sigma}\left[A^{2}-4(\sigma Q)^{2 / \sigma}\right]
$$

that $\varphi$ takes reasonable value. 


\section{CONCLUSION}

We obtained the regular particle-like solutions to the scalar field equations with induced nonlinearity in external gravitational fields described by Freedman-RobertsonWalker and Gödel Universes respectively. Beside the usual solitons, a special type of regular localized configurations, known as droplets, have been obtained. It has been shown that the droplet-like configurations possess limited energy density and finite total energy and the droplets of different linear sizes up to the soliton share one and the same total energy. It is noteworthy to remark that in the spherically symmetrical case (i.e., in the FRW Universe) at $r_{c} \rightarrow \infty$ for $k=0$ droplet transfers to usual solitonian solution, while for $k= \pm 1$ this is not the case. It has also been shown that in FRW space-time equations with different types of nonlinearities may contain stable solutions, whereas in the case of the Gödel Universe only the droplet-like and hat-like configurations may be stable. Also note that in FRW space-time with $k=+1$ the field function is confined in the region $0 \leq r \leq 1$ independent of the choice of interaction function $P(\varphi)$. Let us note one more additional point of induced nonlinearity. It is well known that time-independent solutions of the equations of scalar field with usual nonlinearity

$$
\left(\frac{\partial^{2}}{\partial t^{2}}-\nabla^{2}\right) \phi+F^{\prime}(\phi)=0
$$

with $F(\phi)$ being the arbitrary function of $\phi$ are unstable [22,23]. But as it has been shown the equations with induced nonlinearity possess particle-like solutions some of which may be stable. Thus we conclude that the "induced nonlinearity" plays the decisive role in formation of stable soliton-like solutions, whereas the external gravitational field can strengthen or weaken the stability.

Acknowledgements The author thanks Prof. G.N. Shikin for useful consultations and Prof. S. Randjbar-Daemi and High Energy Section, AS-ICTP for hospitality. 


\section{REFERENCES}

[1] Scott, A.C., Chu, F.Y.F., and McLaughlin, D.W. (1973). Proc. IEEE 61, 1443.

[2] Dodd, R. K., Eilbeck, J.C., Gibbon, J.D., and Morris, H.C. (1982). Solitons and nonlinear wave equations (London, Academic Press).

[3] Perring, J.K. and Skyrme, T.H.R. (1962). Nuclear Physics 31, 550.

[4] Rybakov Yu.P. (1985). Particle Structure in Nonlinear Field Theory (Moscow, PFU).

[5] Rajaraman, R. (1982). Solitons and Instantons: An introduction to Solitons and Instantons in Quantum Field Theory (North Holland Publ. Corp., New York).

[6] Bogoliubov, N.N. and Shirkov, D.V. (1976). An Introduction to the Theory of Quantized Fields (Nauka, Moscow).

[7] Rybakov, Yu.P., Saha, B., and Shikin, G.N. (1997). International Journal of Theoretical Physics 36, 1475.

[8] Marshak, R.E. and Sudershan, E.C.G. (1961). Introduction to Elementary Particle Physics (Int. Sci. Pub. Inc., New York).

[9] Bronnikov, K.A., Melnikov, V.N., Shikin, G.N., and Staniukovich, K.P. (1979). Annals of Physics 118, 84.

[10] Weinberg, S. (1972). Gravitation and Cosmology (John Wiley and Sons, Inc., New York).

[11] Birrell, N.D., and Davies, P.C. (1982). Quantum Fields in Curved Space (Cambridge University Press, London).

[12] Einstein, A. (1917). Sitz. Preuss. Akad. Wiss. 142.

[13] Bronnikov, K.A., Rybakov, Yu.P., and Shikin, G.N. (1991). Izvestia VUZov, Physics 34(5), 24.

[14] Bronnikov, K.A. and Shikin G.N. (1977). Izvestia VUZov, Physics 20(9), 25; (1991). Itogi Nauki i Techniki: Classicheskaya teorya polya i teorya gravitatsii $\mathbf{2}, 4$.

[15] Shikin, G.N. (1995). Theory of Solitons in General Relativity (URSS, Moscow).

[16] Saha, B. (1998). The Bangladesh Journal of Astronomical Research 1 (1), 30; (1999). Journal of Theoretical, Mathematical and Computational Physics 2 (1), 15.

[17] Werle, J. (1977). Physics Letters B 71, 367.

[18] Rybakov, Yu.P., Saha, B., and Shikin, G.N. (1994). Communications in Theoretical 
Physics 3, 67; (1998). Gravitation \& Cosmology 4 (2) 114.

[19] Rybakov, Yu.P., Saha, B., and Shikin, G.N. (1995). JINR Preprint E2-95-197.

[20] Kamke, E. (1959). Differentialgleichungen Lösungsmethoden und Lösungen (Leipzig)

[21] Gödel, A. (1949). Review Modern Physics 21, 447.

[22] Hobart, R.H. (1993). Proceedings of Physical Society 82, 201.

[23] Derrick, G.H. (1964). Journal of Mathematical Physics 5, 1252. 


\section{FIGURES}

FIG. 1. Perspective view of interaction function $P(\varphi)$ providing (anti-)droplet like configurations for different values of $\sigma$. Here (and later on) the thick-line, dash-line, dash-dot-line and thin-line correspond to the value of $\sigma=3,5,7,9$ respectively.

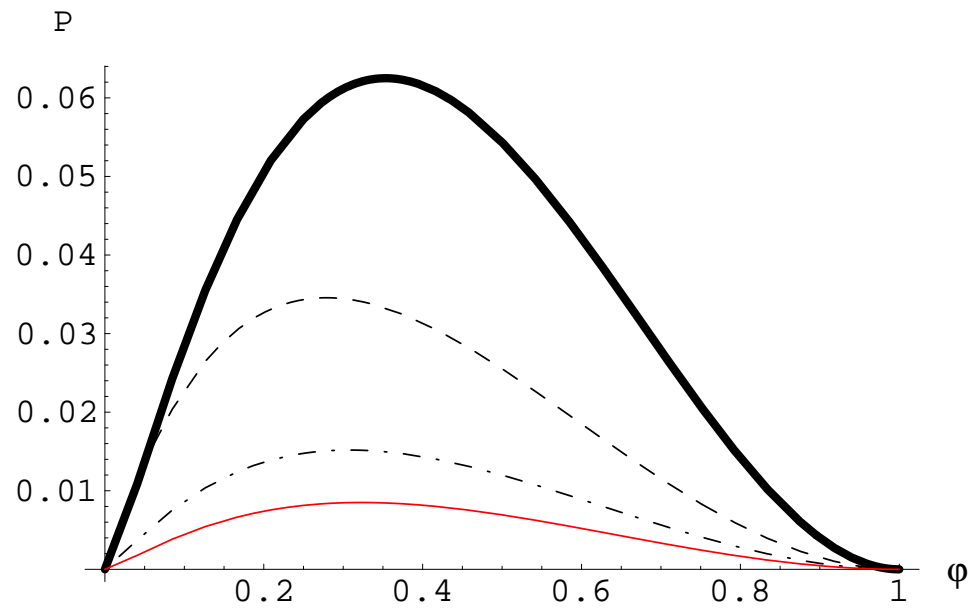


FIG. 2. Perspective view of droplet-like configurations. As one sees, $\varphi$ is completely located in the region $0 \leq r \leq r_{c}$.

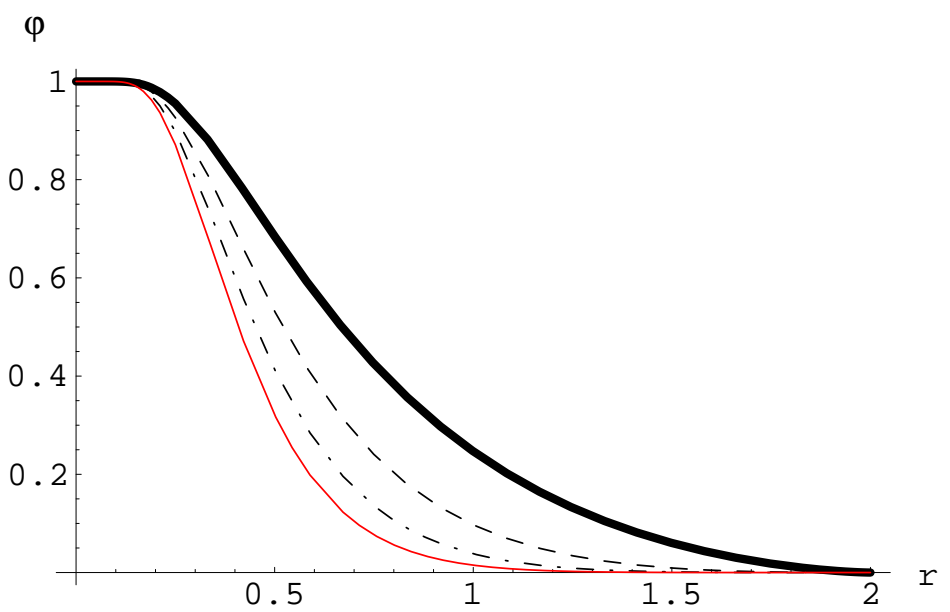




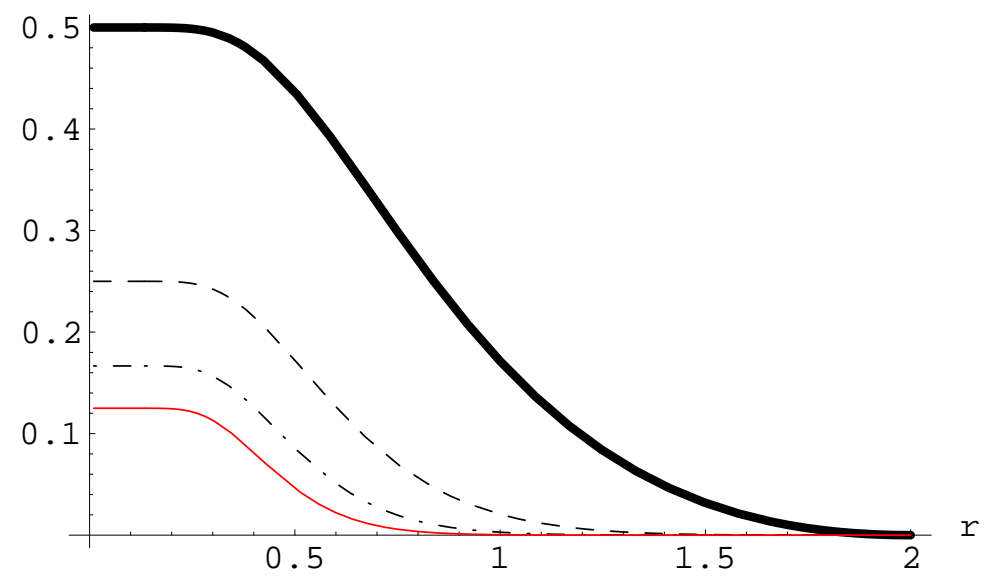

FIG. 3. Perspective view of electromagnetic field $A_{0}$ corresponding to the interaction (3.22). As it comes out, the electromagnetic field also has droplet-like configuration

FIG. 4. Perspective view of the hat-like configurations. As one sees, the configuration possesses nontrivial value only in the interval $(a, b)$.

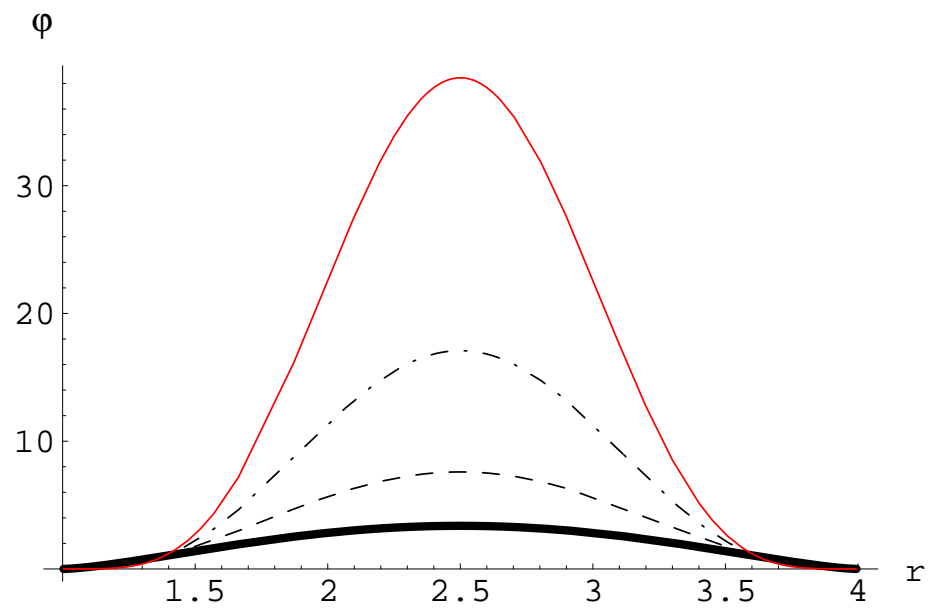

